\title{
Emergence of Extensively Drug-resistant Shigella sonnei in Bangladesh
}

\author{
Mahbubur Rahman*, AKM Fahmidul Haque, Iztiba Mallik Deeba, Dilruba Ahmed, Tanha Zahidi, \\ Afrina Hossain Rimu, Mahmuda Akter, Fatema Akter, K A Talukder
}

International Centre for Diarrhoeal Disease Research, Bangladesh

Copyright $\bigcirc 2017$ by authors, all rights reserved. Authors agree that this article remains permanently open access under the terms of the Creative Commons Attribution License 4.0 International License

\begin{abstract}
The objective of the study was to investigate current species distribution and growing antimicrobial resistance (AMR) of Shigella isolates for proper treatment. Shigellae, isolated from faecal samples in International Centre for Diarrhoeal Disease research, Bangladesh, Dhaka hospital in 2015, were tested for antimicrobial susceptibility by disc diffusion method to ampicillin, co-trimoxazole, ciprofloxacin, azithromycin, mecillinam, ceftriaxone/cefixime and meropenem. Extensively drug-resistant (XDR, resistant to 5 or 6 of 7 useful anti-Shigella drugs tested) Shigella isolates resistant to 6 drugs were analyzed for ESBL and AmpC phenotypes, plasmid profiles, R-plasmids transfer, bla $a_{\mathrm{SHV}}, b l a_{\mathrm{TEM}}$, bla $_{\mathrm{CTX}-\mathrm{M}}, b l a_{\mathrm{OXA}}$ and $m p h \mathrm{~A}, m p h \mathrm{~B}, \operatorname{erm} \mathrm{A}, \operatorname{erm} \mathrm{B}, \operatorname{erm} \mathrm{C}$, ere $\mathrm{A}$, ere $\mathrm{B}, m e f \mathrm{~A}$ and $m s r \mathrm{~A}$ genes by PCR; and clonality of S. sonnei by PFGE. Of 134 isolates cultured from 3722 (3.6\%) diarrhoeal faecal samples, $46 \%$ were $S$. sonnei, $37 \%$ S. flexneri, $4 \% \quad$ S. boydii, $5 \% \quad S$. dysenteriae and $7 \%$ non-typeable. Multidrug-resistant (MDR, resistant simultaneously to $\geq 3$ drugs) $S$. sonnei were $95 \%$ compared to $66 \%(\mathrm{P}<0.01)$ MDR $S$. flexneri including 18\% \& 14\% XDR types, respectively. All isolates were susceptible to meropenem. Four (6\%) S. sonnei, 2 (4\%) S. flexneri and 1 (17\%) S. boydii (total of 7 isolates) were six-drugs XDR; 5 of them had ESBL phenotypes. Three $S$. sonnei and $1 S$. flexneri had bla $a_{\mathrm{TEM}}$ and bla $a_{\mathrm{CTX}-\mathrm{M}} ; 1 S$. boydii had bla $a_{\mathrm{SHV}}$ bla $a_{\mathrm{TEM}}$ and bla $a_{\text {CTX-м}} ; 1 S$. sonnei had bla $a_{\text {TEM }} \beta$-lactamase. All but one $S$. flexneri had only mphA gene on 62-MDa conjugative-R-plasmid coding azithromycin resistance. PFGE identified MDR-S. sonnei Global III clade. Thus, MDR-S. sonnei replaced $S$. flexneri as predominant isolate in Dhaka, Bangladesh; many emerged as XDR strains requiring treatment by meropenem. The findings demand judicial use of antibiotics to contain emergence and spread of resistance locally and globally. Physicians should be informed about MDR and XDR Shigella for judicious prescribing of antimicrobial therapy.
\end{abstract}

Keywords Shigella, Antimicrobial Resistance, ESBL, mphA, Azithromycin

\section{Introduction}

Shigella is one of the significant causes of diarrhoeal diseases globally with $\sim 80$ million cases and $\sim 700,000$ deaths/year, mostly occurring in developing countries [1]. Of the four species of genus Shigella, Shigella flexneri and Shigella sonnei predominate. S. sonnei, which has historically been prevalent in developed countries, is currently undergoing an unprecedented expansion across industrializing regions in Asia, Latin America, and the Middle East replacing $S$. flexneri as the major species [2]. The current evolutionary changes in temporal distribution of Shigella species across the globe have altered the epidemiology, treatment and preventive strategies of bacillary dysentery (shigellosis). Antimicrobial therapy has been recommended for patients with shigellosis because it can limit the clinical course of illness and reduce the risk of complications and the duration of faecal excretion of the causative organism, reducing the spread of infection $[1,3]$. The therapy also improves the growth and nutritional status of affected children, especially in developing countries [3]. A major problem, however, is the increasing resistance of Shigella spp. to useful antimicrobial agents [2-5]. Over the decades, Shigella isolates resistant to multiple first-line antimicrobial agents, such as sulphonamides, tetracycline, ampicillin, trimethoprim-sulphamethoxazole (SXT) and nalidixic acid have been reported from many countries including Bangladesh [2-5] resulting in difficulties in the selection of empirical therapy. Currently ciprofloxacin is recommended as the drug of choice by the World Health Organization for the therapy of first-line drug-resistant Shigella infections in both adults and children [1]. In addition, ceftriaxone, pivmecillinam (amdinocillin pivoxil) and azithromycin are considered as alternative drugs [1]. However, multidrug-resistant (MDR; resistant to three or more classes of antimicrobial agents) Shigella isolates have been reported worldwide and few reliable treatment options 
exist, particularly in developing countries. The recent acquisition of ciprofloxacin-resistance and/or extended-spectrum-cephalosporin-resistance and/or azithromycin-nonsusceptibility by the MDR Shigella isolates further narrows the choice of effective antimicrobial agents for treating shigellosis [4-5].

Recently, multidrug-resistant $S$. sonnei has emerged as a serious public health threat globally [6-10]. In South and South-East Asia, and elsewhere multidrug-resistant S. sonnei has become highly prevalent and now partially/completely replaced $S$. flexneri probably as a part of global extension of MDR-S. sonnei Global III clade [4, 6-12]. International travel or recent migration of refugees from endemic areas of Asia and Africa has accelerated the global spread of drug-resistant Shigella to non-endemic countries by repeated introductions and autochthonous transmission $[5,7,14,15]$. Recently MDR-S. sonnei resistant to ciprofloxacin with decreased susceptibility to azithromycin (MIC $>16 \mu \mathrm{g} / \mathrm{ml}$ ) caused widespread outbreaks in the USA $[5,14]$ resulting in difficulties in selection of antimicrobial agents for proper treatment. Shigella species, resistant to ciprofloxacin, ceftriaxone and azithromycin (MIC $>32 \mu \mathrm{g} / \mathrm{ml}$ ) caused infections in refugees and local residents in Austria and other EU countries imposing a new health problem [15]. Even the last resource meropenem was used to treat ceftriaxone-resistant Shigella infection because MIC values of meropenem (MIC range $<0.06 \mu \mathrm{g} / \mathrm{ml}$ ) and imipenem (MIC range $<0.06-0.25 \mu \mathrm{g} / \mathrm{ml}$ ) showed susceptibility of all resistant Shigella isolates tested [16, 17]. In Bangladesh, during 2001-2002 S. sonnei comprised only $16 \%$ of 266 Shigella isolates studied and a low resistance to ampicillin $(16 \%)$ and high resistance to cotrimoxazole $(87 \%)$ were reported among $S$. sonnei isolates [3]. However, the prevalence of $S$. sonnei increased from $7.2 \%$ in 2001 to $25 \%$ in 2011in Bangladesh [12]. In Bangladesh ESBL-mediated ceftriaxone-resistant MDR-S. sonnei was reported in 2004 [13] and ciprofloxacin-resistant MDR-S. sonnei in 2013 [12]. Currently, we do not have sufficient information on the prevalent serotypes and susceptibility patterns of Shigella isolates to many useful antimicrobial agents, such as mecillinam, ciprofloxacin, azithromycin,

ceftriaxone/cefixime and meropenem. Given the impact of suboptimal use of antimicrobial agents in Bangladesh and the ability of Shigella to develop resistance after the introduction of new antimicrobial agents for treatment, it is not unlikely that antimicrobial resistance patterns in Bangladesh have changed since they were reported in the past [11-13]. Thus, the continuing changing patterns of prevalent species and resistance of Shigella isolates indicate the need for monitoring antimicrobial susceptibility. We aimed to study the current changing patterns of Shigella species and their antimicrobial resistance to explore the impact of global changes in Shigella infections and for determination of optimal antimicrobial therapy for shigellosis in Bangladesh.

\section{Materials and Methods}

\subsection{Study Design, Bacterial Isolates and Antimicrobial Susceptibility Testing}

During June-December, 2015, we studied consecutive Shigella isolates from Clinical Microbiology Laboratory, International Centre for Diarrhoeal Disease Research, Bangladesh (icddr,b) Dhaka, Bangladesh. The centre's Dhaka hospital is located in the capital Dhaka that serves $\sim 150,000$ dairrhoeal patients per year from all over Bangladesh. The faecal samples of diarrheal patients were cultured and isolates were identified and serotyped by standard methods in Clinical Microbiology Laboratory [18]. Antimicrobial susceptibility to ampicillin, cotrimoxazole, ciprofloxacin, azithromycin, mecillinam,

ceftriaxone/cefixime and meropenem was carried out by the disc diffusion method using CLSI-2014 guidelines except for azithromycin for which British Society of Antimicrobial Chemotherapy (BSAC) guidelines was used $(\mathrm{S} \geq 18 \mathrm{~mm}$, $\mathrm{R}<18 \mathrm{~mm}$ ) $[19,20]$. Minimum inhibitory concentration (MIC) was determined by agar dilution technique and E-test (bioMérieux, France) for selected-resistant isolates only. Extensively drug-resistant (XDR)-Shigella was defined as isolates resistant to 5 or 6 of 7 (ampicillin, cotrimoxazole, ciprofloxacin, azithromycin, mecillinam,

ceftriaxone/cefixime and meropenem) anti-Shigella drugs used for the treatment of shigellosis and tested for in-vitro antimicrobial susceptibility of Shigella in our laboratory. Multidrug-resistant (MDR) Shigella was defined as an isolate simultaneously resistant to $\geq 3$ anti-Shigella drugs. Antibiotics regarded as inappropriate for treatment of shigellosis and rarely tested for in-vitro susceptibility were not considered in defining XDR and MDR isolate. Six (available) XDR-Shigella isolates were further analyzed for MICs of ceftriaxone and azithromycin.

\subsection{Biotyping of $S$. sonnei}

Biotyping of S. sonnei was done using standard methods for fermentation of rhamnose and xylose and hydrolysis of ortho-nitrophenyl-b-Dgalactopyranoside (ONPG), and biotypes were designated according to methods described elsewhere [12].

\subsection{Phenotypic and Genotypic Studies for Ceftriaxone and Azithromycin Resistance}

Six (available) XDR-Shigella isolates were further analyzed for phenotypic and genotypic characteristics of resistance of ceftriaxone and azithromycin. One S. flexneri isolate was not available. The ESBL and AmpC phenotypes were detected by the double disc diffusion synergy test (DDST) and combined disc test (CDT) following CLSI guideline [19]. PCR was carried out for detection of $b l a_{\mathrm{SHV}}$, $b l a_{\mathrm{TEM}}, b l a_{\mathrm{CTX}-\mathrm{M}}, b l a_{\mathrm{OXA}}$ for cetriaxone resistance and $m p h \mathrm{~A}$, $m p h \mathrm{~B}$, erm $\mathrm{A}, \operatorname{erm} \mathrm{B}, \operatorname{erm} \mathrm{C}$, ere $\mathrm{A}$, ere $\mathrm{B}, m e f \mathrm{~A}$ and $m s r \mathrm{~A}$ gene for azithromycin resistance [21-25]. The primer sequences used in PCR were mentioned in Table 1[21-25]. 
Table 1. Oligonucleotide primers used for the detection of Shigella sonnei ESBL and macrolide resistance genes by PCR in the study [21-25].

\begin{tabular}{|c|c|c|c|c|}
\hline Target gene & Primer & Sequence, $5^{\prime} \rightarrow 3^{\prime}$ & $\begin{array}{l}\text { Annealing temperature } \\
{ }^{0} \mathrm{C}\end{array}$ & Product size, bp \\
\hline \multirow[t]{2}{*}{${ }^{B l a}$ TEM } & TEM-F & СTTCCTGTTTTTGCTCACCCA & 52 & 717 \\
\hline & TEM-R & TACGATACGGGAGGGCTTAC & & \\
\hline \multirow[t]{2}{*}{${ }^{B l a}$ SHV } & SHV-F & TCAGCGAAAAACACCTTG & 52 & 471 \\
\hline & SHV-R & TCCCGCAGATAAATCACC & & \\
\hline \multirow[t]{2}{*}{${ }^{B l a}$ OXA } & OXA-F & ACCAGATTCAACTTTCAA & 55 & 598 \\
\hline & OXA-R & TCTTGGCTTTTATGCTTG & & \\
\hline \multirow[t]{2}{*}{${ }^{B l a}$ CTX-M } & CTX-M-F & TTTGCGATGTGCAGTACCAGTAA & 51 & 544 \\
\hline & CTX-M-R & CGATATCGTTGGTGGTGCCATA & & \\
\hline \multirow[t]{2}{*}{$m p h(\mathrm{~A})$} & mphA-F & GTGAGGAGGAGCTTCGCGAG & 60 & 403 \\
\hline & mphA-R & TGCCGCAGGACTCGGAGGTC & & \\
\hline \multirow[t]{2}{*}{$m p h(\mathrm{~B})$} & $\mathrm{mphB}-\mathrm{F}$ & GATATTAAACAAGTAATCAGAATAG & 58 & 494 \\
\hline & mphB-R & GCTCTTACTGCATCCATACG & & \\
\hline \multirow[t]{2}{*}{$\operatorname{erm}(\mathrm{A})$} & ermA-F & TCTAAAAAGCATGTAAAAGAAA & 52 & 533 \\
\hline & ermA-R & CGATACTTTTTGTAGTCCTTC & & \\
\hline \multirow[t]{2}{*}{$\operatorname{erm}(\mathrm{B})$} & ermB-F & GAAAAAGTACTCAACCAAATA & 45 & 639 \\
\hline & ermB-R & AATTTAAGTACCGTTACT & & \\
\hline \multirow[t]{2}{*}{$\operatorname{erm}(\mathrm{C})$} & ermC-F & TCAAAACATAATATAGATAAA & 45 & 642 \\
\hline & ermC-R & GCTAATATTGTTTAAATCGTCAAT & & \\
\hline \multirow[t]{2}{*}{$\operatorname{ere}(\mathrm{A})$} & ereA-F & GCCGGTGCTCATGAACTTGAG & 60 & 420 \\
\hline & ereA-R & CGACTCTATTCGATCAGAGGC & & \\
\hline \multirow[t]{2}{*}{$\operatorname{ere}(\mathrm{B})$} & ereB-F & TTGGAGATACCCAGATTGTAG & 55 & 537 \\
\hline & ereB-R & GAGCCATAGCTTCAACGC & & \\
\hline \multirow[t]{2}{*}{$m e f(\mathrm{~A})$} & mefA-F & AGTATCATTAATCACTAGTGC & 54 & 345 \\
\hline & mefA-R & TTCTTCTGGTACTAAAAGTGG & & \\
\hline \multirow[t]{2}{*}{$m s r(\mathrm{~A})$} & msrA-F & GCACTTATTGGGGGTAATGG & 58 & 384 \\
\hline & msrA-R & GTCTATAAGTGCTCTATCGTG & & \\
\hline
\end{tabular}

\subsection{Plasmid Analysis and R-Plasmid Transfer by Conjugation}

Plasmid DNA was prepared according to the alkaline lysis method of Kado and Liu (1981) with some modifications and plasmid sizes were analyzed by using known molecular mass plasmids in E. coli PDK-9, R-1 and V517 in agarose gels [26]. Conjugal transfer of R-plasmids to Escherichia coli K-12 was carried out by the method of Neu et al [27] and lactose-fermenting transconjugants were selected on MacConkey agar containing azithromycin $(32 \mu \mathrm{g} / \mathrm{ml})$. Like wild-type strains, all transconjugants were tested for antimicrobial susceptibility and plasmid profiles. 62-MDa-plasmid was extracted from agarose gel and subjected to PCR for mphA gene detection [24].

\subsection{PFGE}

PFGE of $S$. sonnei isolates was carried out for detection of clonality by XbaI restriction patterns of genomes.
High-molecular-weight genomic DNA was prepared from 6 drugs-XDR $S$. sonnei study isolates (2015), 6 MDR S. sonnei isolates of the year 2001-2010 and 1 S. flexneri isolates and digests with the restriction endonuclease XbaI. Restriction fragment patterns of chromosomal DNA were demonstrated by PFGE. [28].

\section{Results}

\subsection{Bacterial Isolates and Antimicrobial Susceptibility}

Of 3722 stool cultures, 134 (3.6\%) samples were positive for Shigella. S. sonnei were predominant (46\%, 62 isolates) followed by $S$. flexneri 37\% (50), S. boydii 4\% (6), S. dysenteriae 5\% (7), and atypical Shigella 7\% (9). The overall resistance rates were: ampicillin (43\%), co-trimoxazole (79\%), ciprofloxacin (79\%), azithromycin (87\%), mecillinam (16\%), ceftriaxone/cefixime (11\%) and meropenem $(0 \%)$. The antibiotic resistance frequencies of 
co-trimoxazole (98\%), azithromycin (100\%), ciprofloxacin $(97 \%)$, and ceftriaxone (13\%) were higher in S. sonnei compared to those of co-trimoxazole $(60 \%)$, azithromycin $(72 \%)$, ciprofloxacin $(76 \%)$ and ceftriaxone $(10 \%)$ in $S$. flexneri. All (100\%) S. sonnei were simultaneously resistant to two or more drugs compared to $78 \%$ and $73 \%$ in $S$. flexneri and other species $(\mathrm{P}<0.01$, Table 2). MDR phenotypes (Table 2) were detected in $95 \%$ of $S$. sonnei compared to $66 \%$ in $S$. flexneri isolates $(\mathrm{P}<0.01)$. XDR rate was higher in $S$. sonnei than $S$. flexneri and others (18\% vs. $9 \%, \quad \mathrm{P}<0.01)$, respectively similar to 3 -drug resistance patterns (Table 2, 60\%, 30\% and 27\%; $\mathrm{P}<0.01$ ). Two-drug and one-drug resistance patterns were significantly more in other species than $S$. sonnei and $S$. flexneri $(\mathrm{P}<0.01)$. Four $(6 \%)$ of the 62 S. sonnei and 2 of the 50 S. flexneri $(4 \%)$ and 1 of the 6 S. boydii (17\%) were six-drug XDR isolates.

Table 2. Antimicrobial Resistance patterns of Shigella isolates ( $N=134)$, June-December 2015

\begin{tabular}{|c|c|c|c|c|}
\hline \multirow[b]{2}{*}{$\begin{array}{c}\text { Resistance to number of drugs (resistance } \\
\text { patterns) }\end{array}$} & \multicolumn{4}{|c|}{ Resistance patterns } \\
\hline & Types & $\begin{array}{l}\text { S. sonnei }(\mathbf{N}=62) \text {, } \\
\text { No. strains }(\%)\end{array}$ & $\begin{array}{c}\text { S. flexneri } \\
(\mathrm{N}=50), \text { No. } \\
\text { strains }(\%)\end{array}$ & $\begin{array}{c}\text { Other Shigella spp. } \\
(\mathrm{N}=22) \text {, No. strains } \\
(\%)\end{array}$ \\
\hline XDR* strains (Resistance to $6 \& 5$ drugs) & & $11(18)^{\S}$ & $7(14)$ & $2(9)^{\S}$ \\
\hline Resistance to 6 drugs $(X D R)^{*}$ & AAzCMCrS & $4(6)$ & $2(4)$ & $1(5)$ \\
\hline \multirow[t]{7}{*}{ Resistance to 5 drugs $(X D R)^{*}$} & & $7(11)$ & $5(10)$ & $1(5)$ \\
\hline & AAzCMS & $4(6)$ & $2(4)$ & $0(0)$ \\
\hline & AAzCCrS & $1(2)$ & $2(4)$ & $0(0)$ \\
\hline & $\mathrm{AAzCCr}(\mathrm{i})^{\S} \mathrm{S}$ & $1(2)$ & $0(0)$ & $0(0)$ \\
\hline & $\mathrm{AAzCM}(\mathrm{i}) \mathrm{S}$ & $1(2)$ & $0(0)$ & $0(0)$ \\
\hline & $\mathrm{AAzCM}(\mathrm{i}) \mathrm{Cr}$ & $0(0)$ & $1(2)$ & $0(0)$ \\
\hline & $\mathrm{AAzC}(\mathrm{i}) \mathrm{M}(\mathrm{i}) \mathrm{Cr}(\mathrm{i})$ & $0(0)$ & $0(0)$ & $1(5)$ \\
\hline \multirow[t]{11}{*}{ Resistance to 4 drugs** } & & $11(18)$ & $11(22)$ & $3(14)$ \\
\hline & $\mathrm{AAzCS}$ & $4(6)$ & $5(10)$ & $1(5)$ \\
\hline & $\mathrm{A}(\mathrm{i}) \mathrm{AzCS}$ & $4(6)$ & $0(0)$ & $0(0)$ \\
\hline & AAzMS & $1(2)$ & $0(0)$ & $0(0)$ \\
\hline & $\mathrm{AzCCr}(\mathrm{i}) \mathrm{S}$ & $1(2)$ & $0(0)$ & $0(0)$ \\
\hline & $\mathrm{AzC}(\mathrm{i}) \mathrm{Cr}(\mathrm{i}) \mathrm{S}$ & $1(2)$ & $0(0)$ & $0(0)$ \\
\hline & $\mathrm{AAzC}(\mathrm{i}) \mathrm{S}$ & $0(0)$ & $2(4)$ & $1(5)$ \\
\hline & AAzCS(i) & $0(0)$ & $2(4)$ & $0(0)$ \\
\hline & $\mathrm{AAzCM}(\mathrm{i})$ & $0(0)$ & $1(2)$ & $0(0)$ \\
\hline & AC(i)MS & $0(0)$ & $1(2)$ & $0(0)$ \\
\hline & AAzM(i)S & $0(0)$ & $0(0)$ & $1(5)$ \\
\hline \multirow[t]{8}{*}{ Resistance to 3 drugs } & & $37(60)^{\S}$ & $15(30)^{\S}$ & $6(27)^{\S}$ \\
\hline & $\mathrm{AzCS}$ & $32(52)$ & $6(12)$ & $2(9)$ \\
\hline & $\mathrm{AzC}(\mathrm{i}) \mathrm{S}$ & $4(6)$ & $0(0)$ & $1(5)$ \\
\hline & AzCS(i) & $1(2)$ & $1(2)$ & $0(0)$ \\
\hline & $\mathrm{AAzC}$ & $0(0)$ & $3(6)$ & $0(0)$ \\
\hline & $\mathrm{AC}(\mathrm{i}) \mathrm{S}$ & $0(0)$ & $3(6)$ & $1(5)$ \\
\hline & AAzS & $0(0)$ & $2(4)$ & $1(5)$ \\
\hline & AAzM(i) & $0(0)$ & $0(0)$ & $1(5)$ \\
\hline Resistance to 2 drugs & & $3(5)^{\S}$ & $6(12)^{\S}$ & $5(23)^{\S}$ \\
\hline Resistance to 1 drug & & $\mathbf{0}(\boldsymbol{\theta})^{\S}$ & $7(14)^{\S}$ & $6(27)^{\S}$ \\
\hline Susceptible to all drug tested & & $0(0)$ & $4(8)$ & $0(0)$ \\
\hline
\end{tabular}

$\mathrm{A}=$ ampicillin, $\mathrm{Az}=$ azithromycin, $\mathrm{C}=$ ciprofloxacin, $\mathrm{Cr}=$ ceftriaxone, $\mathrm{M}=$ mecillinam, $\mathrm{S}=$ cotrimoxazole. ${ }^{\S} \mathrm{P}<0.01$, *XDR $=$ extensively drug-resistant, overall XDR rate $15 \%,{ }^{\$}(\mathrm{i})=$ intermediate.

†' Others: S. boydii 4\% (6), S. dysenteriae 5\% (7), and atypical Shigella 7\% (9), The overall resistance rates were: ampicillin (43\%), co-trimoxazole (79\%), ciprofloxacin $(79 \%)$, azithromycin $(87 \%)$, mecillinam $(16 \%)$, ceftriaxone/cefixime $(11 \%)$ and meropenem $(0 \%)$. 


\subsection{Biotyping of XDR S. sonnei}

Of 4 XDR $S$. sonnei isolates tested, all were classified as biotype $\mathrm{g}$ (ONPG positive, rhamnose and xylose negative).

\subsection{Phenotypic and Genotypic Studies for Ceftriaxone and Azithromycin Resistance}

Five $(83 \%)$ of 6 six-drug-XDR strains were positive for ESBL by DDST and CDT. Three $S$. sonnei (including one ESBL-phenotype negative $S$. sonnei) and $1 S$. flexneri had bla $_{\mathrm{TEM}}$ and $b l a_{\mathrm{CTX}-\mathrm{M}} ; S$. boydii had bla $a_{\mathrm{SHV}}, b_{\mathrm{TEM}}$ and $b l a_{\text {CTX-M }}$; the rest $S$. sonnei had only $b l a_{\text {TEM }}$ (Fig 2 a)

\subsection{Plasmid Analysis and R-Plasmid Transfer by Conjugation}

Most Shigella isolates had 3-4 plasmids (Fig. 1; 140, 62, 2.7, 2.1MDa). Only 62-MDa-plasmid carrying azithromycin and ampicillin resistances were transferred from 5 Shigella isolates (excluding $S$. flexneri) to $E$. coli $\mathrm{K}-12$

(transconjugants) by conjugation (data not shown). A 403bp PCR amplicon obtained by using gel-eluted 62-MDa-plasmid DNA indicated the presence of $m p h$ A gene in 5 of 6 XDR Shigella (except S. flexneri) isolates (Fig. 2 b).

\subsection{PFGE}

XbaI restriction fragment patterns of DNA from three (S1, S2, S6) of four XDR S. sonnei study isolates and 3 (A1, A2 and A3) reference $S$. sonnei pulsotype A isolates were identical with minor diversifications (Fig. 3). XbaI produced approximately 21 fragments, except one isolate (S5) which produced 18 fragments showing a different pulsotype of biotype $\mathrm{g} /$ global III clade. All the strains were typeable that yield reproducible banding patterns. Three (S1, S3 and S6) of 4 study $S$. sonnei isolates belonged to major pulsotype A of $S$. sonnei [12] by PFGE with minor variants of A pulsotype (95\% or more similarity) and belonged to biotype g group indicating Global III clade [6, 12].

$$
\begin{array}{llllllllll}
1 & 2 & 3 & 4 & 5 & 6 & 7 & 8 & 9 & 10
\end{array}
$$

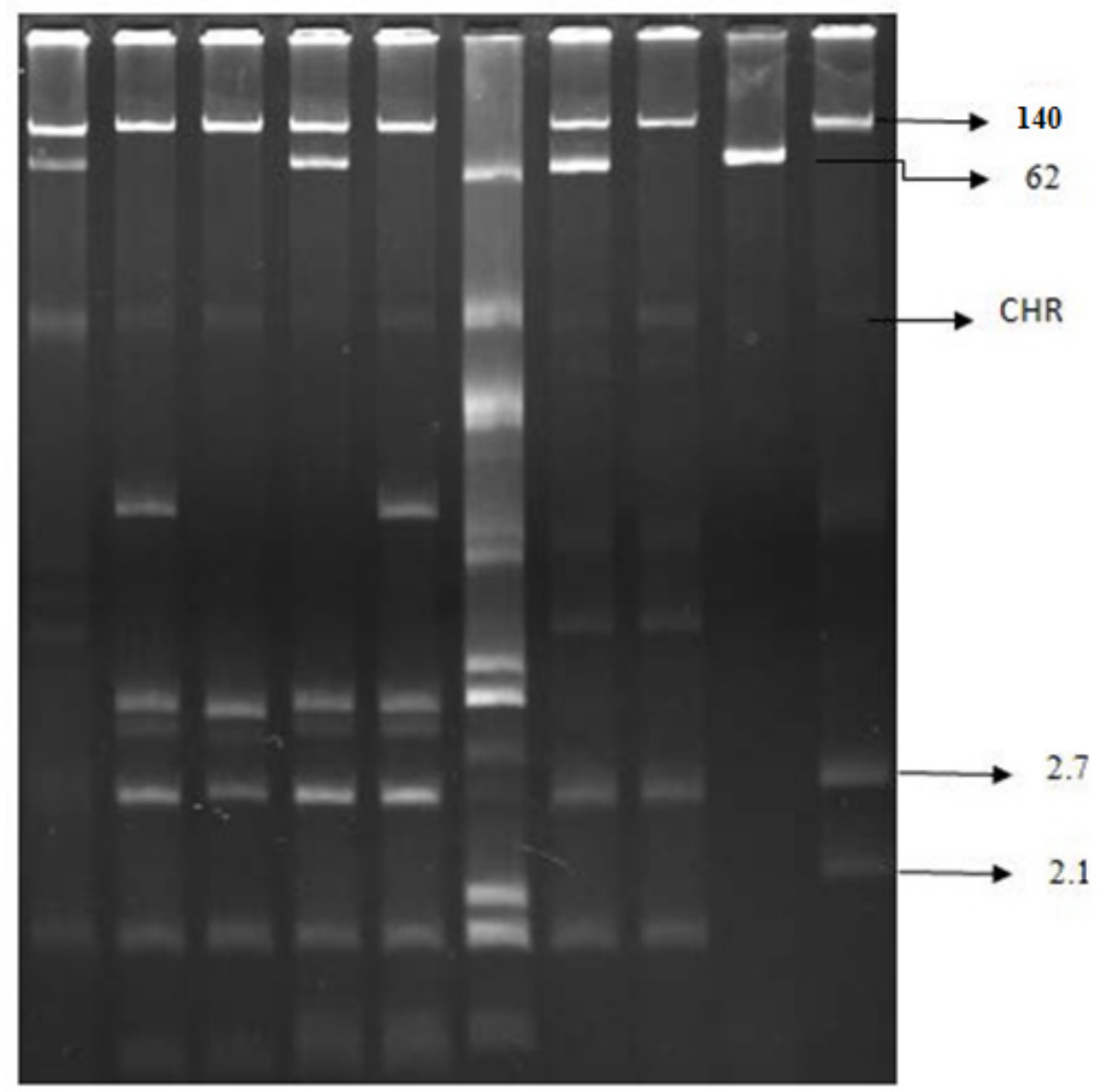

Figure 1. Agarose gel electrophoresis of plasmid DNA (MDa) showing representative patterns of Shigella boydii and Shigella sonnei isolates. Lane: 1, 4 $\left(\mathrm{Azm}^{\mathrm{R}}\right.$ S. sonnei $\mathrm{S} 1$ and S3); Lane: 2, 3, 5 ( Azm $^{\mathrm{S}}$ S. sonnei); Lane: 6 E. coli V-517; Lane 7 (Azm ${ }^{\mathrm{R}}$ S. boydii B4); Lane 8 (Azm ${ }^{\mathrm{S}}$ S. boydii); Lane: 9 E. coli R-1; Lane: 10 PDK-9. 
(a)

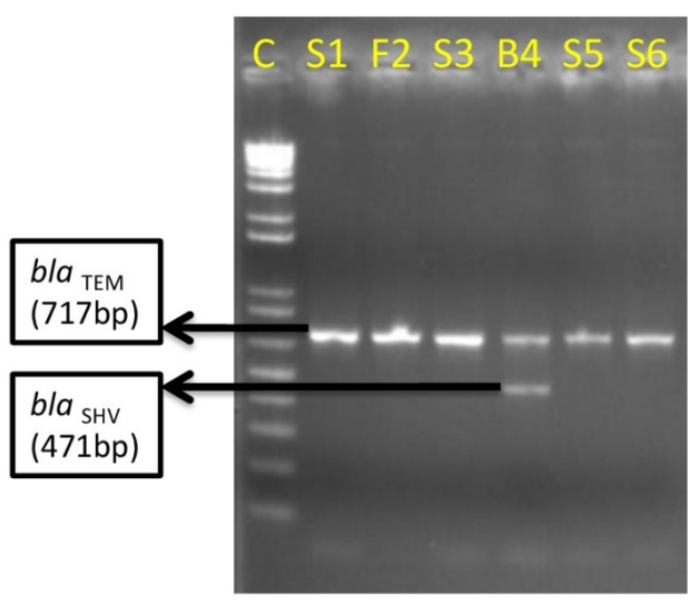

(b)

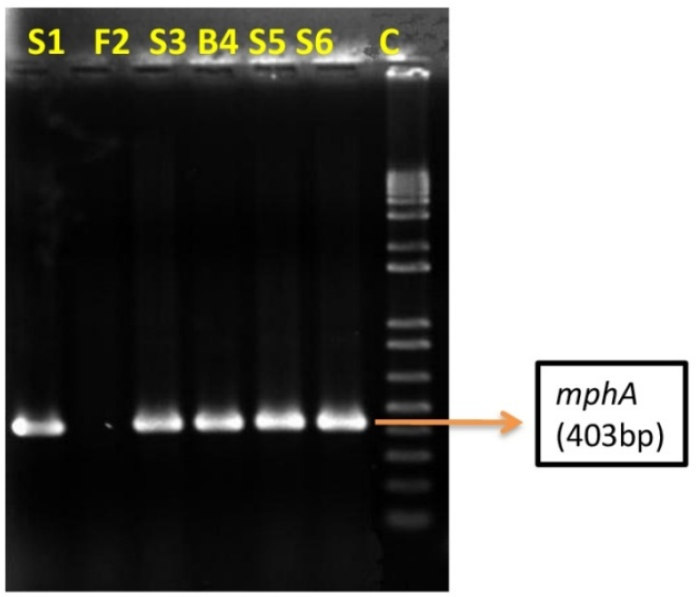

Figure 2. Agarose gel of PCR results showing $b l a_{\mathrm{TEM}}$ and $b l a_{\mathrm{SHV}}$ (gel a) and $m p h \mathrm{~A}$ genes (gel b) of six XDR Shigella isolates

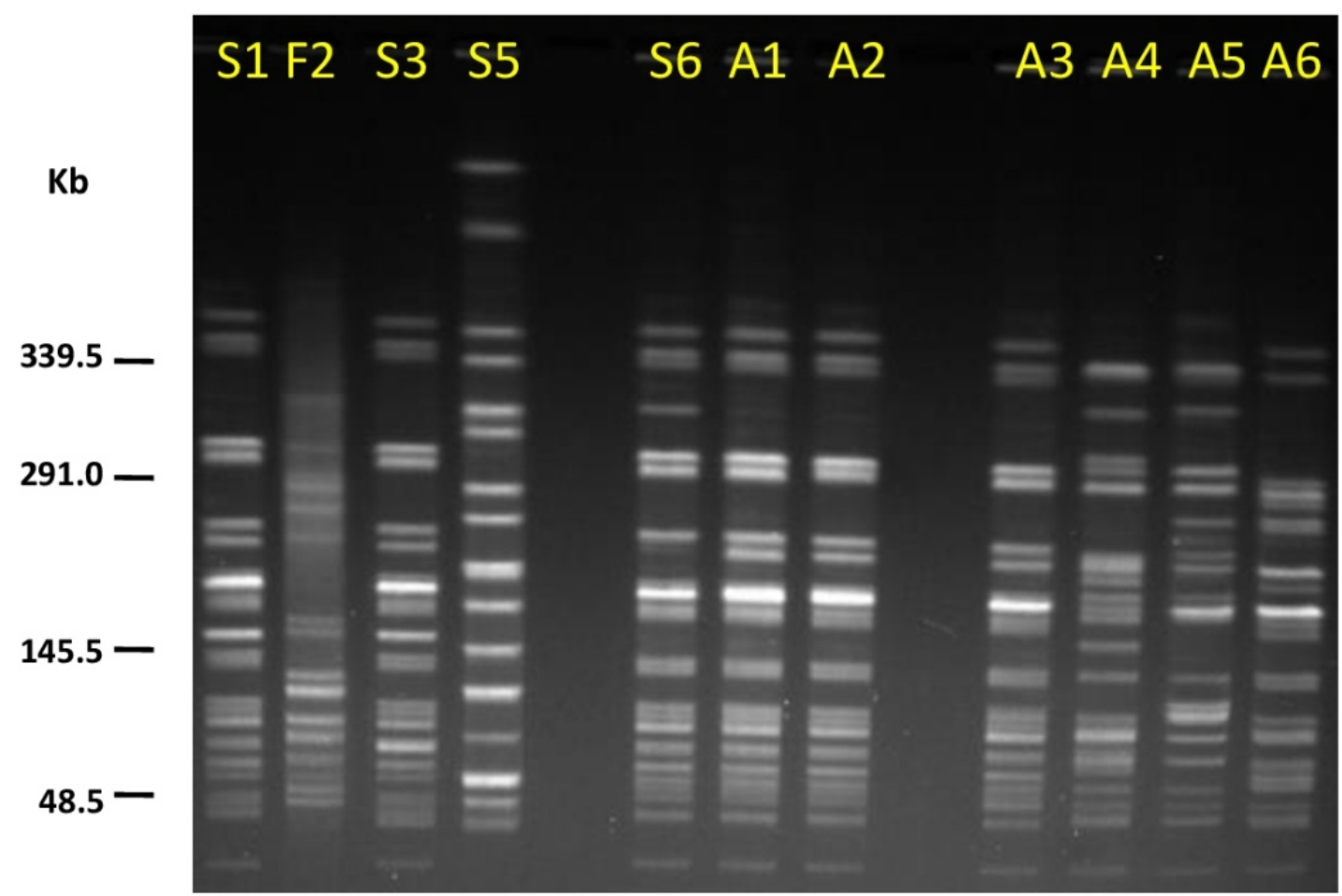

Figure 3. PFGE results showing XbaI-digested chromosomal DNA patterns of XDR Shigella sonnei. S. sonnei: Lane S1, S3, S6 belonged to pulsotype A, Lane S5 belonged to new pulsotype. Lane F2 S. flexneri; Reference $S$. sonnei strains (12): Lane A1, A2 and A3: pulsotype A; Lane A4 and A5: pulsotype C and A6: pulsotype B $S$. sonnei isolates were used for comparison of PFGE results. 


\section{Discussion}

S. sonnei has been primarily responsible for bacillary dysentery in developed countries. At present it is undergoing an unprecedented spread across Asia, America, and the Middle East which represents an emerging threat to public health globally $[2,7]$. S. flexneri was the most prevalent species of Shigella in 2011 in Bangladesh [11]. However, an increase in the isolation rate $S$. sonnei in Dhaka and Mirpur was noted during 2010-2011 [11, 12]. We documented in the present study that $S$. sonnei replaced $S$. flexneri as the predominant isolate in Dhaka for the first time in 2015. Biotype $\mathrm{g}$ of $S$. sonnei is a marker of MDR Global III clade [6, $7,12]$. Biotype g of $S$. sonnei was found to comprise major PFGE pulsotype A with minor diversifications (B, C and D types) [12]. We identified six-drugs-XDR four $S$. sonnei biotype g MDR Global III clade isolates that belonged to A pulsotype/clone ( 3 isolates) and its variants by PFGE (a descendant of MDR Global III clade) indicating the emergence, adaptation and dissemination of XDR S. sonnei Global III clade for the first time in Bangladesh [6, 12].

Multidrug resistance was a problem in $S$. flexneri and $S$. dysenteriae in Bangladesh which was less common in $S$. sonnei $[3,13]$. However, the highest prevalence of XDR as well as MDR phenotypes was observed in S. sonnei in Dhaka, Bangladesh in our study. In 2004, we reported S. sonnei isolates that were resistant to numerous drugs and produced R-plasmid mediated ESBL [13] which appeared to acquire from $E$. coli in the gut [29]. Five drugs-XDR-patterns were highly prevalent in all Shigella species being susceptible to ceftriaxone, or mecillinam along with meropenem. Six drugs-XDR $S$. sonnei can only be treated with meropenem or imipenem. Resembling Vietnam, adaptive genomic microevolution in $S$. sonnei might have occurred in Bangladesh over the last few years [6] and S. sonnei acquired resistance to many drugs by mutation and lateral gene transfers to emerge as a key resistant species. In the USA, $\mathrm{XDR}$ and multiply resistant $S$. sonnei was reported to cause dysentery outbreaks in 34 states resulting in 315 cases during 2014-2015, making treatment increasingly difficult $[5,14]$. XDR-S. sonnei was also reported to cause infections among refugees and local residents in Austria and many other EU countries in 2015 [15].

Extended-spectrum $\beta$-lactamase was detected in ceftriaxone-resistant Shigella by phenotypic characteristics in Bangladesh [13]. We detected multiple ESBL genes in a single Shigella strain for the first time in Bangladesh that will lead all third-generation cephalosporin ineffective for the treatment of shigellosis. Azithromycin is effective for the treatment of ciprofloxacin-resistant shigellosis both in children and adults. The $m p h \mathrm{~A}$ gene encodes a macrolide 2'-phosphotransferase that phosphorylates and inactivates azithromycin (macrolides) is spreading azithromycin-resistance in Europe and USA [14, 24]. The majority $(83 \%)$ of XDR-Shigella carry R-plasmid mediated $m p h \mathrm{~A}$ gene. The conjugal transfer of $m p h \mathrm{~A}$ plasmid from
Shigella to E. coli K-12 indicates the potential of spread of azithromycin-resistance to other bacteria in near future in Bangladesh.

Our findings have major implications for dissemination of XDR-S. sonnei and other species regionally and globally, and for spread of mobile drug-resistance genes among bacterial populations. Options for antimicrobial therapy for such XDR-Shigella infections are very limited that clearly demands judicial use of antibiotics, development of vaccines, safe food and water, improvement of hygiene and sanitation for the prevention and control of shigellosis.

In conclusion, physicians should be warned regarding the presence of XDR and high prevalence of MDR Shigella spp., particularly XDR-S. sonnei Global III clade in Dhaka that might be prevalent in other parts of Bangladesh. Further studies might explore the extent of spread in the country.

Monitoring of antimicrobial susceptibility of clinical isolates for optimum antimicrobial therapy is essential. Additionally, reduced susceptibility of Shigella strains to azithromycin and mecillinam should be identified by MICs determination for the early detection of the treatment failures and emergence of resistance. When indicated, pivmecillinam or ceftriaxone might be considered for treating shigellosis in Bangladesh with caution. Meropenem may be used as the last resort.

\section{Acknowledgements}

This research study was funded by core donors which provide unrestricted support to icddr, b for its operations and research. Current donors providing unrestricted support include: Government of the People's Republic of Bangladesh; the Department of Foreign Affairs, Trade and Development (DFATD), Canada; Swedish International Development Cooperation Agency (Sida) and the Department for International Development (UK Aid). We gratefully acknowledge these donors for their support and commitment to International Centre for Diarrhoeal Disease Research, Bangladesh (icddr,b) Dhaka, Bangladesh research efforts.

\section{Funding}

This research work was funded by Swedish International Development Cooperation Agency (Sida), Grant no.GR01014.

\section{Competing Interests}

None to declare

\section{Ethical Approval}

The Ethical Review Committee of IRB of icddr,b 
approved this research protocol (PR-14042) on August 21, 2014.

\section{REFERENCES}

[1] World Health Organization. Guidelines for the control of shigellosis, including epidemics due to Shigella dysenteriae type 1 [Internet]. Geneva: WHO; 2005. Available from: http://apps.who.int/iris/bitstream/10665/43252/1/924159330 X. pdf?ua $=1 \& u a=1$.

[2] C. N. Thompson, P. T. Duy, S. Baker. The rising dominance of Shigella sonnei: an intercontinental shift in the etiology of bacillary dysentery. PLoS Negl Trop Dis. 9(6), e0003708-e0003708, 2015.

[3] M. Rahman, S. Shoma, H.Rashid, S.E. Arifeen, A. H. Baqui, A. K. Siddique, G. B. Nair, D. A. Sack. Increasing Spectrum in Antimicrobial Resistance of Shigella Isolates in Bangladesh: Resistance to Azithromycin and Ceftriaxone and Decreased Susceptibility to Ciprofloxacin. J HEALTH POPUL NUTR. 25(2):158-67, 2007.

[4] D. Bhattacharya, H. Bhattacharya, D. S. Sayi, A. P. Bharadwaj, M. Singhania, A. P. Sugunan, S. Roy. Changing patterns and widening of antibiotic resistance in Shigella spp. over a decade (2000-2011), Andaman Islands, India. Epidemiology and infection.143:470-7, 2015.

[5] Centers for Disease Control and Prevention. Ciprofloxacin-and azithromycin-nonsusceptible shigellosis in the United States. CDC Health Alert Network. June 4, 2015.

[6] K.E. Holt, T.V.T. Nga, D.P. Thanh, H. Vinh, D.W. Kim, M.P.V. Tra, J. I. Campbell et al. Tracking the establishment of local endemic populations of an emergent enteric pathogen. Proceedings of the National Academy of Sciences, 110(43), 17522-17527, 2013.

[7] K. E. Holt, S Baker, FX Weill, EC Holmes, A Kitchen et.al. Shigella sonnei genome sequencing and phylogenetic analysis indicate recent global dissemination from Europe. Nature genetics,44(9), 1056-1059, 2012.

[8] X.P. Koh, C.S. Chiou, N. Ajam, H. Watanabe, N. Ahmad, K. L. Thong. Characterization of Shigella sonnei in Malaysia, an increasingly prevalent etiologic agent of local shigellosis cases. BMC Infectious Diseases, 12(1), 2012.

[9] S. Ruekit, S. Wangchuk, T. Dorji, K.P. Tshering, P. Pootong, P. Nobthai, O. Serichantalergs, K. Poramathikul, L. Bodhidatta, C.J.Mason. Molecular characterization and PCR-based replicon typing of multidrug resistant Shigella sonnei isolates from an outbreak in Thimphu, Bhutan. BMC research notes, 7(1), p.1, 2014.

[10] S. Qiu, X. Xuebin, Y. Chaojie, W. Jian, L. Beibei, L. Peng, L. Hao et al. Shift in serotype distribution of Shigella species in China, 2003-2013. Clinical Microbiology and Infection, 21(3), pp.252-e5, 2015.

[11] S.K. Das, S. Ahmed, F. Ferdous, F. D. Farzana, M.J. Chisti, D. T. Leung, M.A. Malek et al. Changing emergence of Shigella sero-groups in Bangladesh: observation from four different diarrheal disease hospitals. PloS one, 8(4), p.e62029, 2013.

[12] A.I. Ud-Din, S.U.Wahid, H.A. Latif, M. Shahnaij, M. Akter, I. J. Azmi, T. N. Hasan et al. Changing trends in the prevalence of Shigella species: emergence of multi-drug resistant Shigella sonnei biotype $\mathrm{g}$ in Bangladesh. PloS one, 8(12), p. e82601, 2013. doi:10.1371/journal.pone.0082601.

[13] M. Rahman, S. Shoma, H. Rashid, A. K. Siddique, G. B. Nair, D. A. Sack. Extended-spectrum $\beta$-lactamase-mediated third-generation cephalosporin resistance in Shigella isolates in Bangladesh. Journal of Antimicrobial Chemotherapy, 54(4), pp.846-847, 2004.

[14] A. Bowen, J. Hurd, C. Hoover, Y. Khachadourian, E. Traphagen, E. Harvey, T. Libby, et al. Importation and domestic transmission of Shigella sonnei resistant to ciprofloxacin-United States, May 2014-February 2015. MMWR Morb Mortal Wkly Rep, 64(12), pp.318-20, 2015.

[15] I. Lederer, K. Taus, F. Allerberger, S. Fenkart, A. Spina, B. Springer, D. Schmid. Shigellosis in refugees, Austria, July to November 2015. Euro Surveill, 20(48), p.30081, 2015. DOI:http://dx.doi.org/10.2807/1560-917.ES.2015.20.48. 30081

[16] N. Goel, C. Wattal, D. Kaul, V. K. Khanna. Emergence of ceftriaxone resistant Shigella. The Indian Journal of Pediatrics, 80(1), pp.70-71, 2013.

[17] R.N. Jones, H. K. Huynh, D. J. Biedenbach, T.R. Fritsche, H. S. Sader. Doripenem (S-4661), a novel carbapenem: comparative activity against contemporary pathogens including bactericidal action and preliminary in vitro methods evaluations. Journal of Antimicrobial Chemotherapy, 54(1), pp.144-154, 2004.

[18] P.R. Murray, K. S. Rosenthal, M.A. Pfaller. Medical microbiology. Elsevier Health Sciences, 2015.

[19] P. A. Wayne. Clinical and laboratory standards institute. Performance standards for antimicrobial susceptibility testing 17, 2007.

[20] British society for antimicrobial chemotherapy. BSAC Methods for Antimicrobial Susceptibility Testing, Version 12, May 2013.

[21] P. Lal, A. Kapil, B. K. Das, S. Sood. Occurrence of TEM \& SHV gene in extended spectrum [beta]-lactamases (ESBLs) producing Klebsiella sp. isolated from a tertiary care hospital. Indian Journal of Medical Research, 125(2), p.173, 2007.

[22] Islam, S.S., Malek, M.A., Haque, A.F., Talukder, K.A. and Akhter, M.Z. Beta Lactamase Genes of Extended Spectrum Beta Lactamase Producing Escherichia coli from Anorectal Sepsis Cases in Bangladesh. Bangladesh Journal of Microbiology, 30(1-2), pp.23-29, 2016.

[23] MCP Nguyen, PL Woerther, M. Bouvet, A. Andremont, R. Leclercq and A. Canu. Escherichia coli as reservoir for macrolide resistance genes. Emerg Infect Dis, 15(10), pp. 1648-1650. 2009.

[24] L. Boumghar-Bourtchai, P. Mariani-Kurkdjian, E. Bingen, I. Filliol, A. Dhalluin, S. A. Ifrane, F.X. Weill, R. Leclercq. Macrolide-resistant Shigella sonnei. Emerg Infect Dis, 14(8), pp.1297-9, 2008.

[25] M. Edelstein, M. Pimkin, I. Palagin, I. Edelstein, and L. Stratchounski. Prevalence and molecular epidemiology of 
CTX-M extended-spectrum $\quad \beta$-lactamase-producing Escherichia coli and Klebsiella pneumoniae in Russian hospitals. Antimicrobial agents and chemotherapy, 47(12), pp.3724-3732. 2003.

[26] C. A. Kado, S. T. Liu. Rapid procedure for detection and isolation of large and small plasmids. Journal of bacteriology, 145(3), pp.1365-1373, 1981.

[27] H. C. Neu, C. E. Cherubin, E. D. Longo, B. Flouton, J. Winter. Antimicrobial resistance and R-factor transfer among isolates of Salmonella in the northeastern United States: a comparison of human and animal isolates. Journal of Infectious Diseases, 132(6), pp.617-622, 1975.

[28] E. M. Ribot, M. A. Fair, R. Gautom, D. N. Cameron, S. B. Hunter, B. Swaminathan, T. J. Barrett. Standardization of pulsed-field gel electrophoresis protocols for the subtyping of Escherichia coli O157: H7, Salmonella, and Shigella for PulseNet. Foodbourne Pathogens \& Disease, 3(1), pp.59-67, 2006. doi: 10.1089/fpd.2006.3.59.

[29] H. Rashid, M. Rahman. Possible transfer of plasmid mediated third generation cephalosporin resistance between Escherichia coli and Shigella sonnei in the human gut. Infection, Genetics and Evolution, 30, pp.15-18, 2015. 